OPEN ACCESS

Edited by:

Murugesan Velayutham, West Virginia University, United States

Reviewed by:

Hou-Zao Chen,

Chinese Academy of Medical Sciences and Peking Union Medical

College, China

Leonardo Yuji Tanaka,

Universidade de São Paulo, Brazil

*Correspondence:

Luciano Gattinoni

gattinoniluciano@gmail.com

${ }^{+}$Present address:

Serge Masson,

Roche Diagnostics, Rotkreuz,

Switzerland

Specialty section: This article was submitted to

Redox Physiology, a section of the journal

Frontiers in Physiology

Received: 22 March 2021

Accepted: 07 July 2021

Published: 06 August 2021

Citation:

Bonifazi M, Meessen J, Pérez A, Vasques $F$, Busana $M$, Vassalli $F$, Novelli D, Bernasconi R, Signori C,

Masson S, Romitti F, Giosa L,

Macri M, Pasticci I, Palumbo MM,

Mota F, Costa M, Caironi P, Latini R,

Quintel M and Gattinoni L (2021)

Albumin Oxidation Status in

Sepsis Patients Treated With

Albumin or Crystalloids.

Front. Physiol. 12:682877.

doi: 10.3389/fphys.2021.682877

\section{Albumin Oxidation Status in Sepsis Patients Treated With Albumin or Crystalloids}

\author{
Matteo Bonifazi', Jennifer Meessen², Alba Pérez ${ }^{3}$, Francesco Vasques', Mattia Busana', \\ Francesco Vassalli', Deborah Novelli ${ }^{2}$, Roberto Bernasconi ${ }^{2}$, Chiara Signori ${ }^{2}$, \\ Serge Masson ${ }^{2 \dagger}$, Federica Romitti', Lorenzo Giosa', Matteo Macrì', lacopo Pasticci', \\ Maria Michela Palumbo ${ }^{1}$, Francisco Mota ${ }^{4}$, Montserrat Costa ${ }^{3}$, Pietro Caironi ${ }^{5,6}$, \\ Roberto Latini ${ }^{2}$, Michael Quintel ${ }^{1}$ and Luciano Gattinoni ${ }^{\text {** }}$
}

${ }^{1}$ Department of Anaesthesiology, Emergency and Intensive Care Medicine, University of Goettingen, Göttingen, Germany, ${ }^{2}$ Department of Cardiovascular Medicine, Istituto di Ricerche Farmacologiche Mario Negri IRCCS, Milan, Italy, ${ }^{3}$ Bioscience Research Group, Grifols, Barcelona, Spain, ${ }^{4}$ Medical Affairs, Grifols, Barcelona, Spain, ${ }^{5}$ Department of Anaesthesia and Critical Care, AOU "S. Luigi Gonzaga, Turin, Italy, ${ }^{6}$ Department of Oncology, University of Turin, Turin, Italy

Inflammation and oxidative stress characterize sepsis and determine its severity. In this study, we investigated the relationship between albumin oxidation and sepsis severity in a selected cohort of patients from the Albumin Italian Outcome Study (ALBIOS). A retrospective analysis was conducted on the oxidation forms of human albumin [human mercaptoalbumin (HMA), human non-mercapto-albumin form 1 (HNA1) and human non-mercaptoalbumin form 2 (HNA2)] in 60 patients with severe sepsis or septic shock and 21 healthy controls. The sepsis patients were randomized (1:1) to treatment with 20\% albumin and crystalloid solution or crystalloid solution alone. The alloumin oxidation forms were measured at day 1 and day 7 . To assess the albumin oxidation forms as a function of oxidative stress, the 60 sepsis patients, regardless of the treatment, were grouped based on baseline sequential organ failure assessment (SOFA) score as surrogate marker of oxidative stress. At day 1, septic patients had significantly lower levels of HMA and higher levels of HNA1 and HNA2 than healthy controls. HMA and HNA1 concentrations were similar in patients treated with albumin or crystalloids at day 1, while HNA2 concentration was significantly greater in albumin-treated patients $(p<0.001)$. On day 7, HMA was significantly higher in albumin-treated patients, while HNA2 significantly increased only in the crystalloids-treated group, reaching values comparable with the albumin group. When pooling the septic patients regardless of treatment, albumin oxidation was similar across all SOFA groups at day 1 , but at day 7 HMA was lower at higher SOFA scores. Mortality rate was independently associated with albumin oxidation levels measured at day 7 (HMA log-rank $=0.027$ and HNA2 log-rank $=0.002$ ), irrespective of treatment group. In adjusted regression analyses for 90-day mortality, this effect remained significant for HMA and HNA2. Our data suggest that the oxidation status of albumin is modified according to the time of exposure to oxidative stress (differences between day 1 and day 7). After 7 days of treatment, lower SOFA scores correlate with higher albumin antioxidant capacity. The trend toward a positive effect of albumin treatment, while not statistically significant, warrants further investigation.

Keywords: albumin, oxidation, sepsis, shock, resuscitation, oxidative stress 


\section{INTRODUCTION}

Human serum albumin, through its cysteine-associated thiol group $(-\mathrm{SH})$ at position 34 , is the main antioxidant compound in the extracellular compartment (Anraku et al., 2013). Albumin continuously circulates from the intravascular compartment to the interstitial space and again into the intravascular compartment through certain capillaries that are permeable to albumin. The half-life of serum albumin is approximately 21 days. During this time, albumin is exposed to the action of free radicals in the extracellular compartment. Therefore, even in normal conditions, albumin may exist in three forms (Colombo et al., 2012; Nakashima et al., 2018):

- Human mercapto-albumin (HMA), in which the thiol groups are present in their reduced form $(-\mathrm{SH})$.

- Human non-mercapto-albumin 1 (HNA1), in which oxidation of the reduced thiol group $(-\mathrm{SH})$ results in a disulfide bond (S-S; this oxidation may be reversible, as the cysteine thiol group may return to its reduced state by regeneration through other antioxidant molecules).

- Human non-mercapto-albumin 2 (HNA2), in which the oxidation of the reduced thiol group $(-\mathrm{SH})$ is irreversible due to binding with sulfonic and sulfinic acid, or to nitrosilation (Colombo et al., 2012) through the reaction of the reduced thiol group $(-\mathrm{SH})$ with nitric oxide.

The extent to which albumin molecules are oxidized by free radicals (reversibly or irreversibly) proportionally decreases its antioxidant capabilities (Matsuyama et al., 2009; Colombo et al., 2012). While there is consistent evidence of the consequences of albumin oxidation in patients with cirrhosis or decompensated hepatic failure (Mera et al., 2005; Arroyo et al., 2014; Das et al., 2017), the literature describing the oxidation status of albumin in sepsis is scant (Quinlan et al., 1998; Colombo et al., 2012). Severe sepsis and septic shock, however, are well-recognized cases of a free radical storm (Huet et al., 2007; Víctor et al., 2009; Galley, 2011; Bavunoglu et al., 2016).

In this study, we investigated the characteristics of albumin oxidation status and the concentrations of several biomarkers in a subset of patients with severe sepsis or septic shock from the Albumin Italian Outcome Study (ALBIOS; Caironi et al., 2014).

\section{MATERIALS AND METHODS}

\section{Study Population}

The study population consisted of a total of 60 patients, 40 with septic shock and 20 with severe sepsis (Singer et al., 2016; Rhodes et al., 2017; Vasques et al., 2018), selected from participants to the ALBIOS study (Caironi et al., 2014). In this multicenter, open-label trial, we randomly assigned 1,818 patients with severe sepsis and septic shock, in 100 intensive care units (ICUs), to receive either 20\% albumin and crystalloid solution or crystalloid solution alone. Patients were randomized (1:1) to treatment with albumin plus crystalloids (referred as albumin-treated group) or treatment with crystalloids alone. Patients were randomly selected for assessment of the albumin redox state from patients with plasma samples available at day 1 and day 7. Selections were balanced for treatment arm and shock. Control samples of plasma $(n=21$, aged $48-80)$ from healthy (not diagnosed with Alzheimer Disease) age-matched subjects were kindly supplied by ACE Foundation - Catalan Institute of Applied Neurosciences (Barcelona, Spain) from their sample repository.

\section{Albumin Oxidation Forms}

The oxidative state of albumin was measured at days 1 and 7 . To assess the redox state of albumin in plasma samples, anionic exchange HPLC (Waters, Milford, MA) was performed using a Shodex-Asahipak ES-502N column (Showa Denko Europe $\mathrm{GmbH}$, Munich, Germany) coupled to a fluorescent detector (Waters) as previously described (Hayashi et al., 2000; Soejima et al., 2004; Oettl and Marsche, 2010). Thawed samples were filtered [0.22 $\mu \mathrm{m}$ pore (Merck Millipore Ltd., Cork, Ireland)] and immediately placed in the carousel of the HPLC apparatus. All the samples were processed in parallel with the controls to ensure sample stability during analysis. Three albumin fractions were identified according to the oxidation status of the thiol group at the Cys34 albumin residue: HMA, HNA1 and HNA2. Percentage was quantified based on peak heights (dividing the peak height of each form by the sum of the peak heights for all albumin forms and multiplying by 100) using Empower software (Waters).

\section{Biomarkers}

Pentraxin 3 (PTX3), presepsin, and chromogranin A (CgA) were assayed at days 1 and 7, as previously described (Mauri et al., 2010; Caironi et al., 2017) as index of the extent of inflammatory response. In addition, N-terminal prohormone of brain natriuretic peptide (NT-proBNP; Masson et al., 2015) was assayed as a marker of volume load and cardiac injury. Redox status was assessed, to document and support the value of sequential organ failure assessment (SOFA) score as global index of the impact of increased oxidative stress on organ function. The following measures were performed: total antioxidant capacity (TAC), myeloperoxidase (MPO), and Malondialdehyde (MDA).

Total antioxidant capacity concentration was measured using the Antioxidant Assay kit (Cayman Chemical, United States, \#709001). The assay is based on the ability of antioxidants in the sample to inhibit oxidation of 2, 2'-azino-di-[3-ethylbenzthiazoline sulfonate] to $\mathrm{ABTS}^{+}$by metmyoglobin. The antioxidants in the sample cause a decrease of the absorbance at $750 \mathrm{~nm}$ and those values are compared with that of Trolox, a water soluble tocopherol analog, and is quantified as $\mathrm{mM}$ Trolox equivalents. MPO was assayed using a colorimetric enzyme-linked immunosorbent assay (ELISA) according to manufacturer's instructions (R\&D Systems, United States). Lipid peroxidation, expressed as MDA, was determined using commercially available kits (MDA Assay Kit, Sigma-Aldrich, Italy, \# MAK085). The method relies on the reaction of MDA, an end product of lipid peroxidation, with thiobarbituric acid producing 
thiobarbituric acid reactive substance, a pink chromogen, which can be measured spectrophotometrically at $532 \mathrm{~nm}$.

\section{Endpoints}

The first endpoint was the difference in the oxidation status of albumin between crystalloids-treated and albumin-treated patients, at day 1 and 7. The secondary endpoint was the assessment of oxidation status of albumin as a function of oxidative stress. In order to investigate the relationship between oxidative stress and the oxidative status of albumin, baseline SOFA score, a surrogate for oxidative stress intensity, was used to stratify patients into three groups (SOFA scores $1-5,6-8$, and 9-13) irrespective of treatment allocation. Oxidative stress, the primary cause of organ failure in sepsis, should be at its peak at baseline compared to the following days.

\section{Statistical Methods}

Baseline clinical characteristics between ALBIOS-treatment arms were compared by means of $\mathrm{Chi}^{2}$, or one-way ANOVA, depending on the nature of the variable. The three albumin forms were expressed as both absolute concentrations $(\mathrm{g} / \mathrm{L})$, and fractions of total concentration. Comparisons were made by means of one-way ANOVA. Biomarkers were compared between treatment groups by means of Mann-Whitney test for non-parametric variables. Additionally, the change of albumin concentrations and fractions measured at day 1 and day 7 was assessed by means of paired Student's $t$-test.

As a surrogate for sepsis severity, patients were divided in three groups by baseline SOFA score (arbitrary cut-off: $1-5$, 6-8, and 9-13), patients characteristics between these groups were compared by means of $\mathrm{Chi}^{2}$ test, one-way ANOVA, or Kruskal-Wallis, as appropriate. Albumin forms at day 1 and day 7 were correlated with the corresponding SOFA scores by means of Pearson or Spearman rank, depending on the distribution of the data.

To assess the association of albumin oxidation forms and 90-day mortality, concentrations of the three albumin-forms were dichotomized using cut-offs estimated from ROC-curves. These dichotomous variables were then included in Kaplan Meier curves and Log Rank analyses. Finally, Cox proportional hazards models were employed (univariately and adjusted for shock and treatment) to assess the independent association of albumin concentration forms and 90-day mortality. Statistical significance was defined as $p<0.05$. Data were analyzed using SPSS Software (IBM SPSS version 25, Armonk NY, United States).

\section{RESULTS}

Baseline clinical characteristics and the most relevant physiological variables of the patient population were wellbalanced between the two ALBIOS treatment arms (Table 1).

\section{Albumin Oxidation and Treatment Arms}

The absolute concentrations of the three forms of albumin (HMA, HNA1, and HNA2) at day 1 are presented in Table 2.
Between the two treatment groups, significant differences were found only in HNA2 concentration at day 1 . Septic patients treated with albumin had higher concentrations of HNA2 as compared to those treated with crystalloids $(3.8 \pm 2.0$ vs. $2.0 \pm 1.4 \mathrm{~g} / \mathrm{L}, p<0.0001)$. On day 1 , HMA concentrations in sepsis patients were significantly reduced in albumin and crystalloids groups compared to healthy controls and to a similar extent. Conversely, the oxidized form HNA2 was significantly increased in both treatment groups compared to healthy controls (Figure 1; Supplementary Table 1).

\section{Time Course of Albumin Oxidation}

As a direct consequence of treatment, patients infused with albumin had significantly higher total albumin (assayed in local laboratories) at day 1 and day 7 compared to crystalloidstreated patients $(p<0.0001)$. As expected, the difference in albumin concentration between groups was larger at day 7 than at day 1 (Table 2).

Human mercapto-albumin and HNA1 concentrations were similar between patient groups on day 1 . However, on day 7 , both forms were significantly higher in those patients who received albumin. HNA2 concentration at both time points was higher in the albumin group (Table 2). Comparing the concentrations from day 1 to day 7 , only the HMA content in the crystalloids-treated group was significantly decreased (from 10.9 to $7.9 \mathrm{~g} / \mathrm{L}, p=0.007$ ). The HNA2 concentration increased in both groups over time. However, the increase was statistically significant only for the crystalloids-treated group (from 2.0 to $2.6 \mathrm{~g} / \mathrm{L}, p=0.01$; Figure 2).

\section{Proportions of Albumin Forms}

In addition to the absolute concentration of the albumin forms, Table 2 presents the percentage of each albumin oxidation form by treatment arm and time point. HNA2 fraction was significantly higher on day 1 but not on day 7 in the albumintreated group compared to the crystalloids group (Day 1: 12.7 vs. $7.7 \%, p=0.002$; Day 7: 13.9 vs. $10.3 \%, p=0.067$ ).

\section{Estimated Oxidative Stress}

The association between baseline SOFA score and the "intensity" of sepsis in this population is shown in Table 3. This table presents the values of biomarkers measured at day 1 and day 7. At day 1, most selected biomarkers were significantly higher in the higher SOFA score group. Lactate, frequency of shock and mortality were increased as well. Also MPO and MDA, as markers of oxidative stress, showed a clear positive trend at day 1, increasing at higher SOFA scores. In contrast, by REDOX status as represented by the different albumin forms (both concentration and proportion) were similar at day 1 in agreement with TAC results. At day 7, HNA1 and HNA2 were higher at higher SOFA scores while HMA showed an inverse trend with SOFA groups. TAC results presented the same tendency as HMA indicating therefore an exhaustion of antioxidant capacity over 7 day exposure to sepsis, see Table 3 . However, due to low $\mathrm{N}$, the results of these analyses were not statistically significant. 
TABLE 1 | Baseline clinical characteristics by randomization treatment.

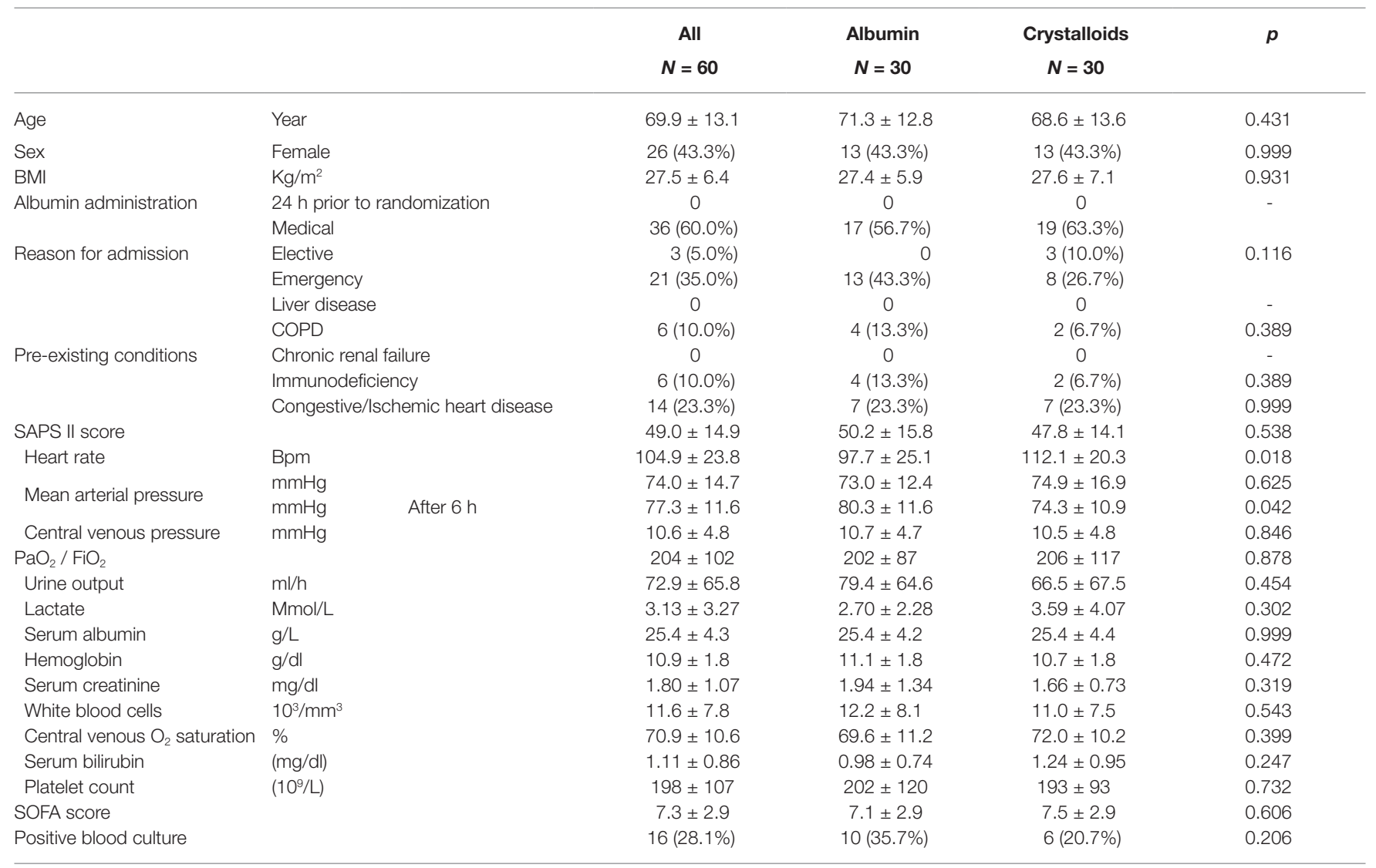

Data are presented as means \pm SD or N (\%). Value of p for one-way ANOVA or Chi between Albumin Italian Outcome Study (ALBIOS) treatment groups. BMI, body mass index; COPD, chronic obstructive pulmonary disease.

TABLE 2 | Forms of albumin by study treatment on day 1 and 7.

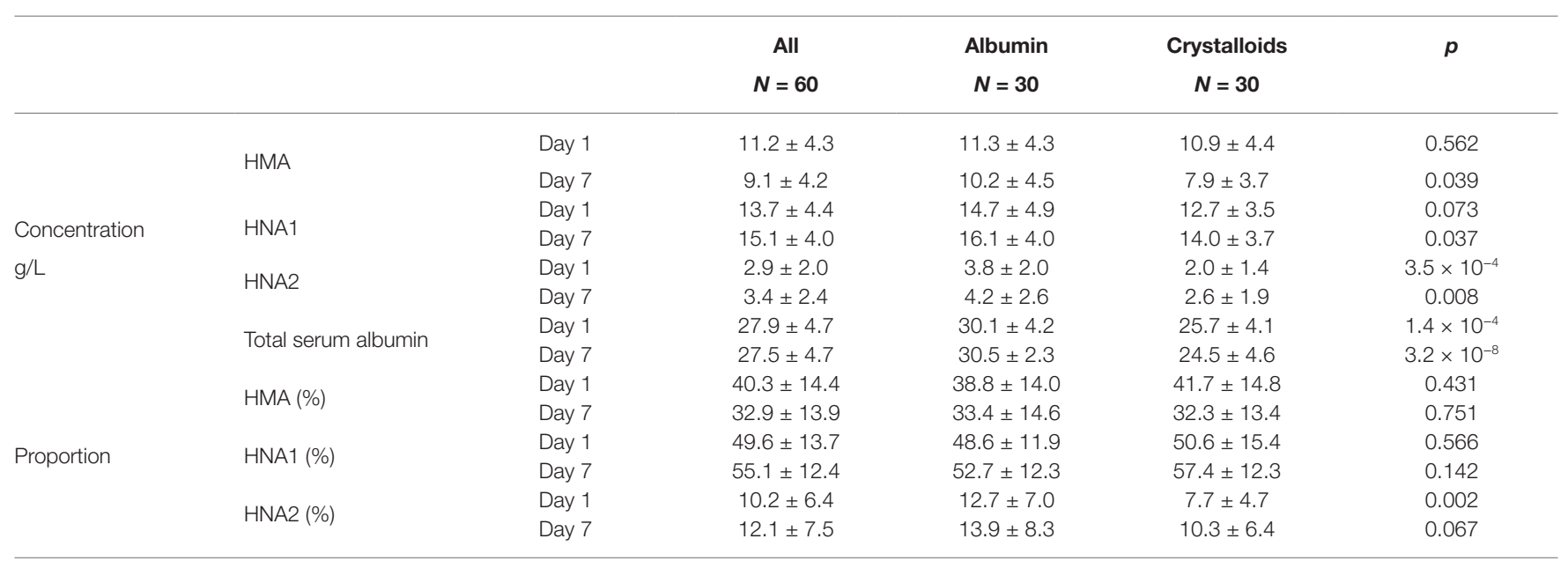

Data are presented as means $\pm S D$. Value of $p$ for one way ANOVA between ALBIOS treatment groups. HMA, human mercaptoalbumin; HNA1, reversibly oxidized human non-mercaptoalbumin; and HNA2, irreversibly oxidized human non-mercaptoalbumin.

\section{Time Course of Oxidative Stress}

At day 7, the SOFA score decreased across the SOFA groups. Expectedly, the SOFA score at day 7 was higher in those who started with a higher SOFA score at baseline $(p<0.0001)$. Most of the biomarkers, except CgA, decreased from day 1 to day 7 , suggesting a decrease in the extent of inflammatory activation and associated oxidative stress in these patients over time. However, the patients in the higher SOFA score group had significantly higher levels of most biomarkers. 


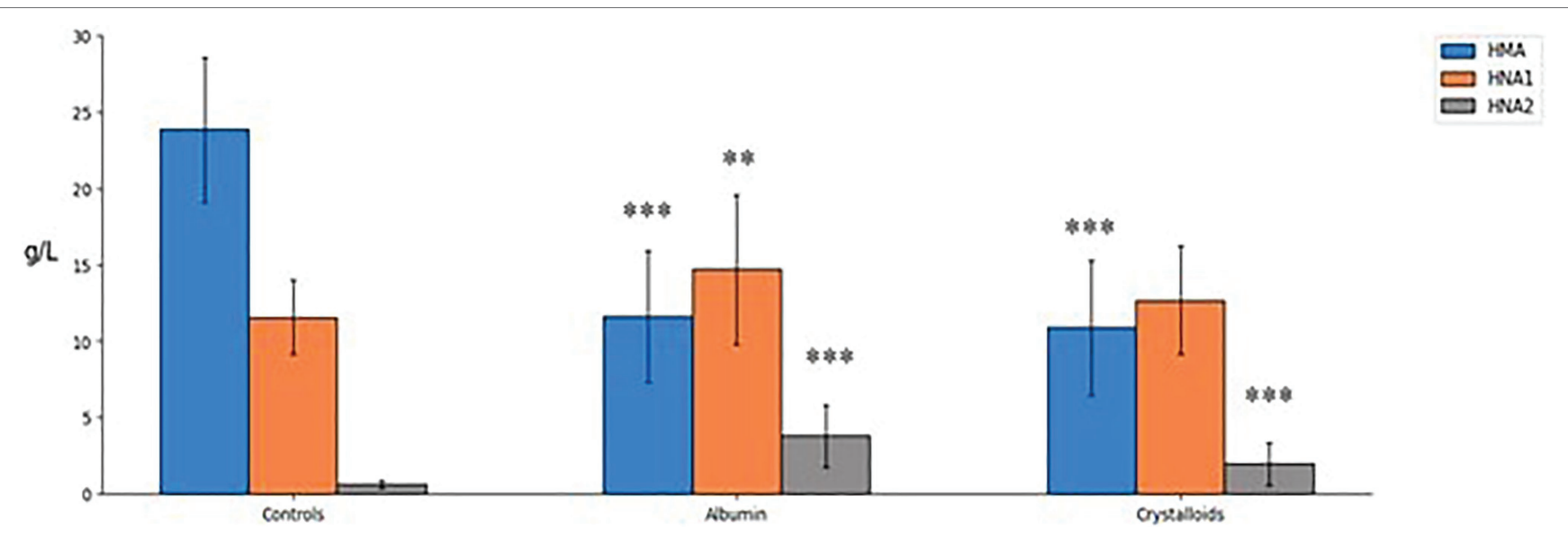

FIGURE 1 | Albumin forms concentration ( $\mathrm{g} / \mathrm{L}$ ) in 21 healthy age-matched controls and 60 septic patients on day 1 . Mean \pm SD, $p$ for treated patients, either albumin or crystalloids vs. healthy control groups (one-way ANOVA): ${ }^{* *} p<0.01$ and ${ }^{* * *} p<0.001$. HMA, human mercaptoalbumin; HNA1, reversibly oxidized human non-mercaptoalbumin; and HNA2, irreversibly oxidized human non-mercaptoalbumin.

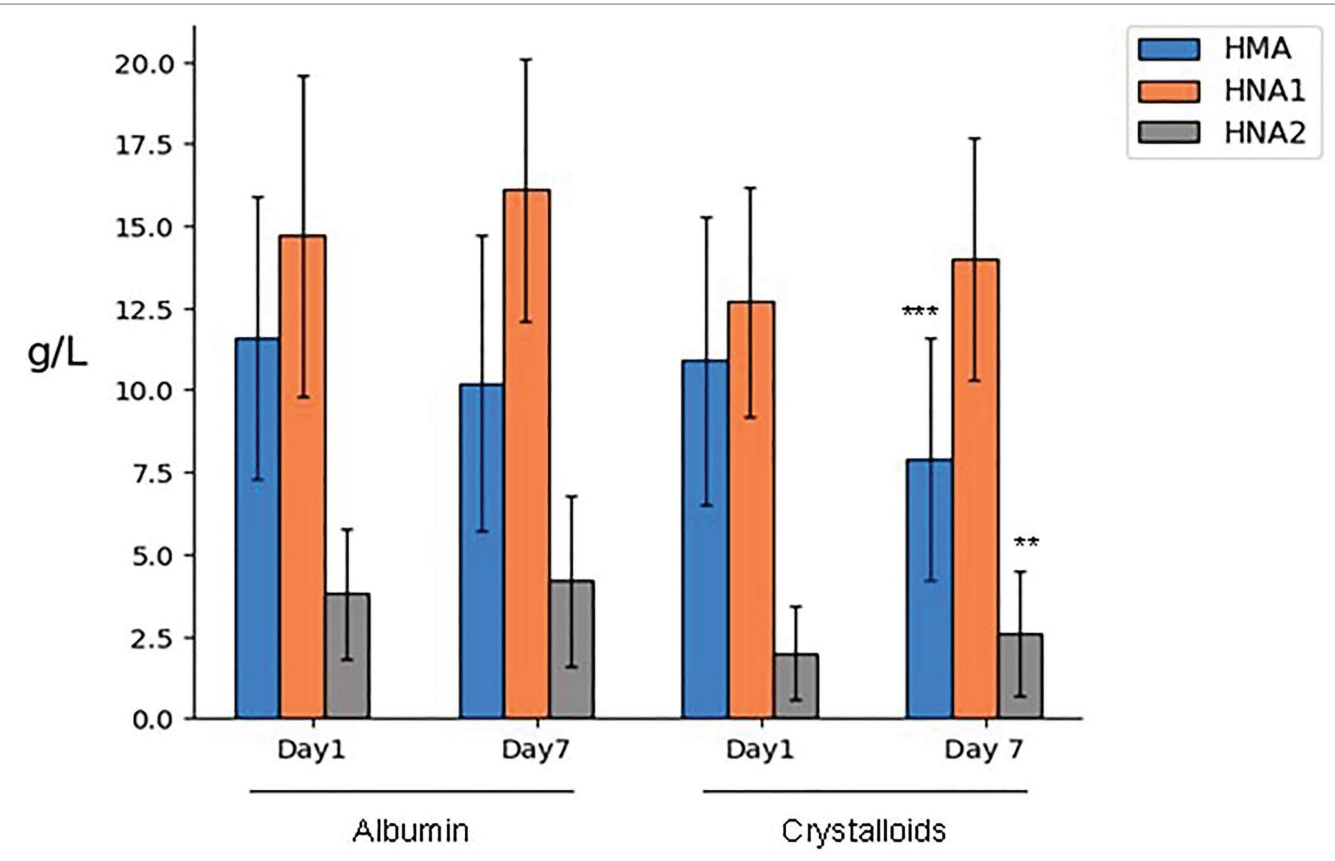

FIGURE 2 | Albumin forms expressed in amounts (g/L) after 1 or 7 days of treatment. Mean \pm SD, $p$ for one-way ANOVA: ${ }^{* *} p<0.01$ and ${ }^{* * *} p<0.001$ day 7 vs. day 1, both for albumin- and crystalloids-treated patients. HMA, human mercaptoalbumin; HNA1, reversibly oxidized human non-mercaptoalbumin; and HNA2, irreversibly oxidized human non-mercaptoalbumin.

Regarding the oxidation status, the decrease of HMA from day 1 to day 7, was larger for patients in the higher SOFAgroup as compared to low-SOFA $(-0.4 \mathrm{~g} / \mathrm{L}$ in low SOFA and -2.9 in high SOFA), while HNA1-concentration had a larger increase in high SOFA-group (+0.1 vs. $+3.0 \mathrm{~g} / \mathrm{L})$. Unexpectedly, HNA2 concentration increase did not differ substantially among baseline SOFA groups, as shown in Table 3. For proportions of the albumin-forms, differences were only found at day 7 with the lowest-SOFA-group having higher percentages of HMA and lower percentages of HNA1. This was further confirmed in a correlation analysis shown in Figure 3. The same trend was visible when limiting the analysis to septic shock patients only, see Supplementary Table 2. When focusing on the 40 patients with septic shock, the trend was even more marked, though it did not reach statistical significance.

\section{Mortality}

The relationship of albumin oxidation forms and mortality was explored by means of ROC curves. Using these curves, the optimal cut-offs for discriminating between surviving and deceased patients were identified: 8.87, 14.99, and $2.32 \mathrm{~g} / \mathrm{L}$ for HMA, HNA1 and HNA2, respectively. Upon creating binary 
TABLE 3 | Circulating biomarkers and albumin oxidative status by baseline sequential organ failure assessment (SOFA) score.

\begin{tabular}{|c|c|c|c|c|c|c|c|}
\hline \multicolumn{3}{|c|}{ Baseline SOFA score* } & $\begin{array}{c}\text { All } \\
N=60^{*}\end{array}$ & $\begin{array}{c}1-5 \\
N=15\end{array}$ & $\begin{array}{c}6-8 \\
N=26\end{array}$ & $\begin{array}{c}9-13 \\
N=17\end{array}$ & $p$ \\
\hline \multirow{3}{*}{\multicolumn{2}{|c|}{ SOFA score }} & baseline & $7.3 \pm 2.9$ & $3.8 \pm 1.4$ & $7.0 \pm 0.8$ & $10.9 \pm 1.2$ & - \\
\hline & & Day 1 & $7.2 \pm 3.6$ & $3.6 \pm 2.2$ & $6.8 \pm 2.3$ & $11.0 \pm 2.3$ & $2.6 \times 10^{-8}$ \\
\hline & & Day 7 & $4.5 \pm 3.2$ & $2.6 \pm 1.4$ & $4.0 \pm 2.2$ & $6.8 \pm 4.1$ & $3.6 \times 10^{-4}$ \\
\hline \multirow{2}{*}{\multicolumn{2}{|c|}{ PTX3 (ng/ml) }} & Day 1 & $57.4(30.8-181.7)$ & $31.9(16.1-84.9)$ & 88.5 (35.8-182.3) & 77.0 (38.4-213.5) & 0.090 \\
\hline & & Day 7 & 19.9 (8.8-34.2) & $12.1(8.5-18.9)$ & $27.5(9.1-37.1)$ & $22.8(8.5-53.8)$ & 0.103 \\
\hline \multirow{2}{*}{\multicolumn{2}{|c|}{ Presepsin (ng/L) }} & Day 1 & $1,160(578-2,142)$ & $514(384-671)$ & $1,160(593-1,849)$ & 2,898 (1824-4,084) & $3.0 \times 10^{-6}$ \\
\hline & & Day 7 & $953(479-2,010)$ & $474(307-1,031)$ & $901(506-1,584)$ & $2016(779-3,211)$ & 0.003 \\
\hline \multirow{2}{*}{\multicolumn{2}{|c|}{ NT proBNP (ng/L) }} & Day 1 & $5,234(1,930-20,044)$ & $1,097(514-4,128)$ & $\begin{array}{c}5,343(2,089- \\
10,484)\end{array}$ & $\begin{array}{c}19,341(5,680- \\
35,000)\end{array}$ & $4.8 \times 10^{-5}$ \\
\hline & & Day 7 & $2050(494-4,576)$ & $336(97-1,196)$ & $1872(517-3,647)$ & $\begin{array}{c}4,314(2,266- \\
15,974)\end{array}$ & $1.0 \times 10^{-4}$ \\
\hline \multirow{2}{*}{\multicolumn{2}{|c|}{$\mathrm{CgA}(\mathrm{pg} / \mathrm{ml})$}} & Day 1 & $211(105-400)$ & $99(67-191)$ & $265(140-414)$ & $253(172-432)$ & 0.011 \\
\hline & & Day 7 & 217 (134-505) & $107(80-184)$ & 242 (163-367) & $335(136-827)$ & 0.007 \\
\hline \multirow{2}{*}{\multicolumn{2}{|c|}{ Lactate (mmol/L) }} & Day 1 & $2.05 \pm 1.51$ & $1.81 \pm 1.62$ & $1.60 \pm 0.69$ & $3.08 \pm 1.98$ & 0.006 \\
\hline & & Day 7 & $1.32 \pm 0.51$ & $1.12 \pm 0.41$ & $1.28 \pm 0.41$ & $1.52 \pm 0.68$ & 0.150 \\
\hline Shock & & & 38 (65.5\%) & 5 (33.3\%) & $17(65.4 \%)$ & $16(94.1 \%)$ & 0.001 \\
\hline \multicolumn{2}{|l|}{90 day mortality } & & 20 (34.5\%) & 2 (13.3\%) & $9(34.6 \%)$ & 9 (52.9\%) & 0.080 \\
\hline \multirow{8}{*}{ Concentration } & \multirow{2}{*}{ HMA (g/L) } & Day 1 & $11.2 \pm 4.3$ & $12.6 \pm 2.9$ & $11.3 \pm 4.5$ & $10.1 \pm 4.4$ & 0.224 \\
\hline & & Day 7 & $9.1 \pm 4.2$ & $12.2 \pm 3.2$ & $8.9 \pm 4.3$ & $7.2 \pm 3.3$ & 0.002 \\
\hline & \multirow{2}{*}{ HNA1 (g/L) } & Day 1 & $13.7 \pm 4.4$ & $14.4 \pm 3.6$ & $13.5 \pm 5.1$ & $13.5 \pm 4.1$ & 0.597 \\
\hline & & Day 7 & $15.1 \pm 4.0$ & $14.5 \pm 3.8$ & $14.4 \pm 4.0$ & $16.5 \pm 4.1$ & 0.209 \\
\hline & \multirow{2}{*}{ HNA2 (g/L) } & Day 1 & $2.9 \pm 2.0$ & $2.3 \pm 1.2$ & $2.6 \pm 1.6$ & $3.3 \pm 2.1$ & 0.322 \\
\hline & & Day 7 & $3.4 \pm 2.4$ & $2.6 \pm 1.5$ & $3.2 \pm 1.8$ & $3.7 \pm 2.2$ & 0.169 \\
\hline & \multirow{2}{*}{ HMA (\%) } & Day 1 & $40.3 \pm 14.4$ & $43.4 \pm 9.6$ & $41.1 \pm 14.7$ & $37.6 \pm 15.9$ & 0.514 \\
\hline & & Day 7 & $32.9 \pm 13.9$ & $42.1 \pm 11.2$ & $33.4 \pm 14.1$ & $26.4 \pm 10.2$ & 0.003 \\
\hline \multirow{4}{*}{ Proportion } & \multirow{2}{*}{ HNA1 (\%) } & Day 1 & $49.6 \pm 13.7$ & $48.8 \pm 8.3$ & $49.6 \pm 15.6$ & $50.9 \pm 15.1$ & 0.926 \\
\hline & & Day 7 & $55.1 \pm 12.4$ & $49.1 \pm 10.4$ & $55.0 \pm 14.2$ & $60.4 \pm 9.4$ & 0.028 \\
\hline & \multirow{2}{*}{ HNA2 (\%) } & Day 1 & $10.2 \pm 6.4$ & $7.7 \pm 4.0$ & $9.3 \pm 4.9$ & $11.6 \pm 6.1$ & 0.126 \\
\hline & & Day 7 & $12.1 \pm 7.5$ & $8.8 \pm 4.6$ & $11.7 \pm 5.9$ & $13.2 \pm 6.3$ & 0.113 \\
\hline \multirow{2}{*}{\multicolumn{2}{|c|}{ TAC (mmol/L) }} & Day 1 & $3.48 \pm 1.06$ & $3.51 \pm 1.01$ & $3.52 \pm 1.21$ & $3.49 \pm 0.94$ & 0.645 \\
\hline & & Day 7 & $3.45 \pm 0.97$ & $3.59 \pm 0.89$ & $3.51 \pm 1.14$ & $3.32 \pm 0.79$ & 0.725 \\
\hline \multirow{2}{*}{ MPO (ng/ml) } & & Day 1 & 105.0 (56.7-282.3) & $72.6(37.4-130.0)$ & 101.8 (67.6-229.3) & 244.7 (116.8-423.8) & 0.042 \\
\hline & & Day 7 & $59.6(39.8-116.0)$ & 50.5 (24.4-97.4) & $53.1(39.4-104.5)$ & 93.5 (42.8-133.5) & 0.371 \\
\hline \multirow{2}{*}{ MDA (nmol/L) } & & Day 1 & $1.67 \pm 2.76$ & $0.98 \pm 0.78$ & $1.26 \pm 1.37$ & $2.94 \pm 4.70$ & 0.082 \\
\hline & & Day 7 & $2.23 \pm 3.28$ & $2.81 \pm 3.99$ & $1.44 \pm 1.68$ & $2.85 \pm 4.41$ & 0.289 \\
\hline
\end{tabular}

Data are presented as means $\pm S D$, median [IQR] or N (\%). Value of p one way ANOVA, Kruskal-Wallis test or Chi ${ }^{2}$ between baseline SOFA score groups. PTX3, pentraxin-3; NTproBNP, NT-pro Brain Naturetic Peptide; CgA, chromogranin A; HMA, human mercaptoalbumin; HNA1, reversibly oxidized human non-mercaptoalbumin; HNA2, irreversibly oxidized human non-mercaptoalbumin; TAC, total antioxidant activity; MPO, myeloperoxidase; and MDA, malondialdehyde.

*Missing SOFA score at baseline for 2 persons.

variables including this cut-off and inserting these in KaplanMeier curves (Table 4), both HMA and HNA2 showed significant effects $(p=0.040$ and $p=0.006)$.

We then included these binary variables in Cox proportional hazard analyses: univariate, lower HMA and higher HNA2 concentrations at day 7 were associated with a significant increase in 90-day mortality [HMA: OR $=3.2$ (95\% CI: $1.2-8.1$ ), $p=0.016$; HNA2: $\mathrm{OR}=0.2(0.0-0.7), p=0.015]$. After adjusting for treatment and shock in the regression analyses, these results remained significant: [HMA: OR=3.9 (95\% CI: 1.3-11.6), $p=0.014 ;$ HNA2: $\mathrm{OR}=0.2(0.0-0.7), p=0.017]$.

\section{DISCUSSION}

In this study, we found: (1) albumin oxidation was far higher in septic patients than in normal subjects; (2) treatment with albumin and crystalloids appeared to be able to prevent the decrease in HMA observed after 7 days of treatment with crystalloids alone; (3) time and level of exposure to oxidative stress, as inferred from the baseline SOFA score, played a substantial role in determining the oxidation status of albumin and (4) in the change of circulating biomarkers; and (5) HMA and HNA2 are in opposing fashion associated with 90-day mortality independent of shock or treatment.

It is well-known that the development of oxidative stress is a distinctive feature of sepsis (Salvemini and Cuzzocrea, 2002). Oxidative stress is defined as the condition in which reactive oxidative species (ROS; free oxygen radicals; Nagar et al., 2018) and other free radicals, such as reactive nitrogen species (RNS; Park and Zmijewski, 2017) sharply increase, challenging the antioxidant defenses. In this condition, ROS originating from mitochondria and from the membrane-bound NADPH oxidase facing the extracellular membrane may damage membranes, cytoplasm and intra-nuclear components. Some ROS, however, may exert their action on extracellular molecules, 
including albumin. This protein may reduce ROS through the oxidation of its thiol-cysteine link group, where three oxidation states of albumin are possible: HMA, HNA1, and HNA2. The redox status of total proteins or the role of other immune proteins were considered marginal in comparison to albumin.
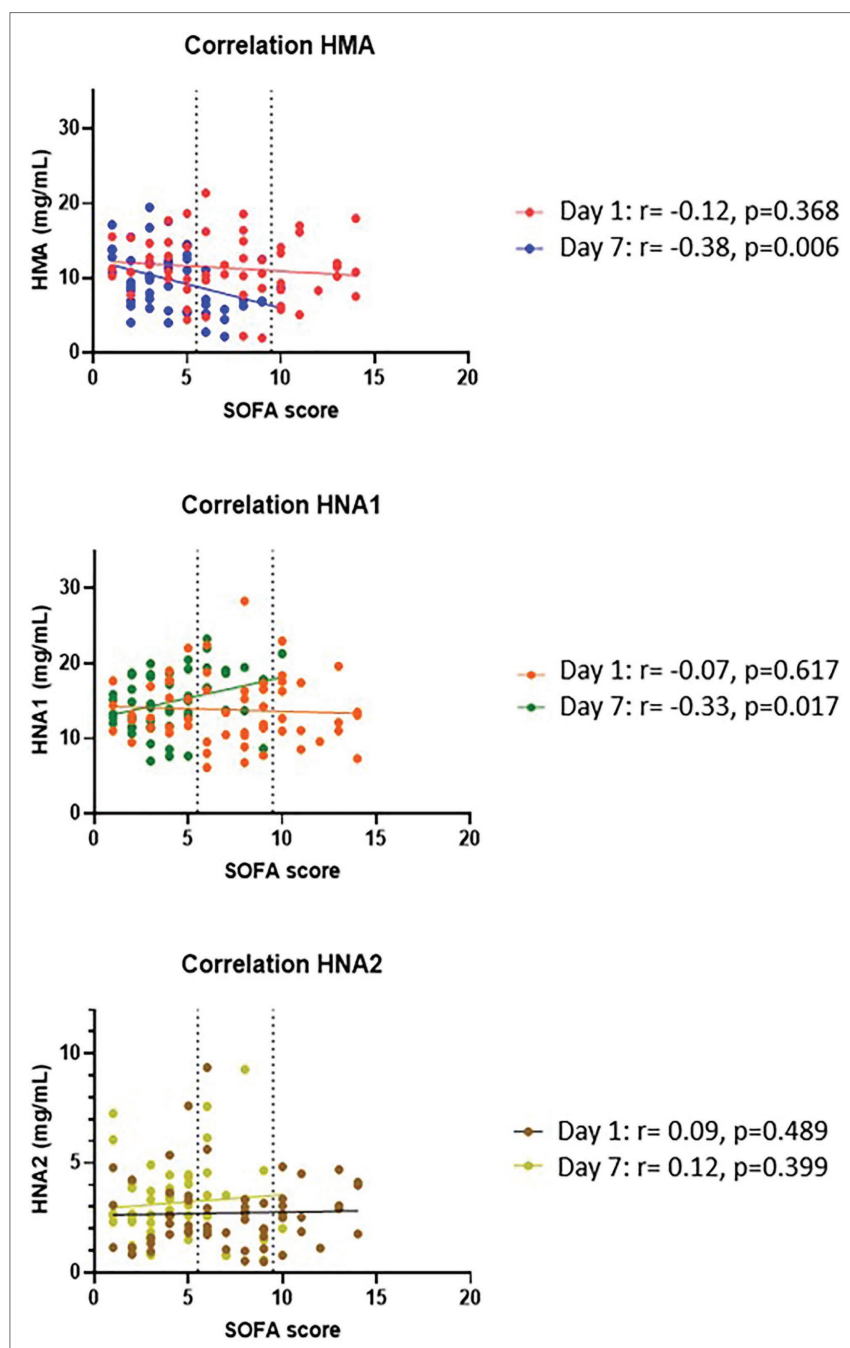

FIGURE 3 | Correlation of albumin oxidation forms and SOFA score. Albumin forms at day 1 and day 7 were correlated with the corresponding SOFA scores by means of Pearson or Spearmen rank, depending on the distribution of the data.
Indeed albumin is the most abundant protein in the human body, with an intravascular (blood plasma) concentration range of $35-52 \mathrm{mg} / \mathrm{ml}$, accounting for $50-60 \%$ of total plasma proteins in healthy humans. Beyond its role as volume expander and transporter, albumin is the main extracellular antioxidant. In addition, albumin concentration is higher than other antioxidant molecules; therefore, albumin would become the main protein in terms of quantities, with antioxidant capacity.

Not surprisingly, during sepsis a ROS storm may quantitatively alter the oxidation status of albumin leading to a decrease in HMA and an increase in HNA1 and HNA2. In this study, we found that after the 1st day of treatment, the fraction of reduced albumin, HMA, and the fraction of reversibly oxidized albumin, HNA1, were similar between patients treated with albumin or crystalloids. Patients infused with albumin had a significantly higher total albumin on day 1 and showed a significant greater concentration of irreversibly oxidized albumin, HNA2. The different concentration of HNA2 could be attributed either to natural processes or a greater amount of irreversibly oxidized albumin fraction in the infused albumin. Considering the similar content of HMA, HNA1 in albumin and crystalloids treated patients at day 1 , it is tempting to say that the difference of HNA2 was due mainly to continuous oxidative stress in the crystalloid arm and to both oxidative stress and to exogenous infused oxidized albumin in the albumin-treated group (Bar-Or et al., 2005).

Despite the observation that modified albumin (i.e., oxidized albumin) can be internalized and degraded by cells (Arroyo et al., 2014), the half-life of albumin, is about 21 days (Vincent et al., 2014). This indicates that most of the molecules of albumin exposed to the ROS storm in the 7-day interval were present throughout the entire interval. When analyzing changes in albumin oxidation status from day 1 to day 7 , only the crystalloids treated patients show a significant decrease in HMA content on top of a significant increase in HNA2. Altogether, these results suggest that patients treated with albumin would show a better evolution of the albumin oxidation profile from day 1 to day 7 than crystalloids-treated patients.

Moreover, we hypothesized that the baseline SOFA score could be used as a surrogate marker for the ROS storm, as the blow phase of sepsis and septic shock is highest in the initial period of the syndrome and declines a few days later (Kumar et al., 2018). Dividing the population into groups by baseline SOFA (Barle et al., 2002; Mantzarlis et al., 2017; Kumar et al., 2018) independent of the treatment, we found that most

TABLE 4 | Regression analyses for day-7 albumin forms and 90-day mortality.

\begin{tabular}{|c|c|c|c|c|c|c|c|c|c|c|c|}
\hline & \multicolumn{3}{|c|}{ ROC analysis } & \multirow{2}{*}{$\begin{array}{l}\text { Optimal } \\
\text { cut-off }\end{array}$} & \multirow{2}{*}{$\frac{\text { Kaplan-Meier }}{\text { Log rank } p}$} & \multicolumn{3}{|c|}{ Univariate Cox regression } & \multicolumn{3}{|c|}{ Adjusted Cox regression* } \\
\hline & AUC & $95 \% \mathrm{Cl}$ & $p$ & & & OR & $95 \% \mathrm{Cl}$ & $p$ & OR & $95 \% \mathrm{Cl}$ & $p$ \\
\hline HMA & 0.66 & $0.51-0.81$ & 0.040 & $8.87 \mathrm{~g} / \mathrm{L}$ & 0.011 & 3.16 & $1.24-8.09$ & 0.016 & 3.90 & $1.31-11.62$ & 0.014 \\
\hline HNA1 & 0.55 & $0.39-0.70$ & 0.562 & $14.99 \mathrm{~g} / \mathrm{L}$ & 0.184 & 0.57 & $0.24-1.33$ & 0.191 & 0.63 & $0.26-1.55$ & 0.313 \\
\hline HNA2 & 0.71 & $0.58-0.85$ & 0.006 & $2.32 \mathrm{~g} / \mathrm{L}$ & 0.005 & 0.16 & $0.04-0.71$ & 0.015 & 0.16 & $0.04-0.72$ & 0.017 \\
\hline
\end{tabular}

Oxidation status was dichotomized based on the optimal cut-off as identified in ROC-analyses. These binary variables were then included in Kaplan-Meier curves as well as Cox proportional hazard models. Reference category for regression analyses is the highest group. HMA, human mercaptoalbumin; HNA1, reversibly oxidized human nonmercaptoalbumin; and HNA2, irreversibly oxidized human non-mercaptoalbumin.

*The adjusted models were adjusted for treatment and shock. 
biomarkers were significantly increased from lowest to highest SOFA groups and these markers declined over the following week. This suggested an improvement over time in the conditions of patients that survived to day 7 .

This supports the hypothesis that the baseline SOFA score was proportional to the intensity of the ROS storm and that this intensity decreased or vanished over 1 week. In support of the assumption that baseline SOFA score reflects intensity of oxidative stress is the higher MDA and MPO concentrations at higher SOFA scores (Table 3). Altogether these data support the close relation between severity of sepsis, intensity of oxidative stress, and organ injury (e.g., SOFA score).

While a positive correlation between biomarker levels and SOFA score may exist, this relationship does not seem to occur between albumin oxidation forms and sepsis severity scores when analyzing pooled patients.

Unexpectedly, albumin oxidation forms were similar at day 1 throughout the SOFA groups. At first, this finding seemed paradoxical. Indeed, when ROS intensity peaked at day 1 , the oxidation of albumin was independent of ROS intensity. However, when the ROS storm had decreased by day 7 , oxidation status was sharply different between patients exposed to higher or lower intensity of ROS storm. In particular, HMA became remarkably different at day 7 indicating an ongoing consumption of the antioxidant body stores after 1 week. This would be more important for patients with higher baseline SOFA scores. In agreement with this observation, a weak correlation between higher HMA values and lower SOFA scores at day 7 was observed. Similarly, TAC as a measure of the total antioxidant capacity, while similar across SOFA scores at day 1, tended to be higher at lower SOFA scores at day 7 .

It is possible that the mechanisms responsible for returning oxidized albumin to its initial reduced status are fully operational at the onset of the syndrome and decrease with time. Furthermore, when separating albumin and crystalloids treatment arms among SOFA groups, after 7 days of treatment, higher percentages of albumin-treated patients ( 80.8 vs. $63.0 \%, p=0.332)$ were found in the group with lowest SOFA scores, Supplementary Table 3. This trend in favor of albumin supplementation may well be due to a higher prevalence of low SOFA score at baseline, 35.7\% in albumin vs. $16.7 \%$ in crystalloids. Although the differences are not statistically significant, this aspect warrants further investigation on a larger number of patients.

Considering all these data, we propose the following model: ROS originating from both the mitochondria and the membranebound NADPH oxidase reach the extracellular matrix, where they randomly oxidize a fraction of circulating albumin. If this occurs, the decrease in the fraction of reduced albumin and the increase in the fraction of oxidized albumin would correlate with the intensity of the ROS storm. On day 1, when antioxidant mechanisms are fully functional, the fractions of albumin are similar suggesting preservation of the capability of the body to reduce albumin. With time, although ROS most likely decline oxidized albumin progressively increases. Due to the long half-life of albumin, the same molecules are repeatedly exposed to oxidative stress. Therefore, the level of oxidized albumin is an indicator of the cumulative oxidative stress that the molecules undergo during the exposure period. Not surprisingly, the mortality rate was found to be associated with the oxidation level of albumin on day 7 , i.e., with the cumulative intensity of oxidative stress. Peak capacity of albumin oxidation was not reached according to the absolute values of HNA2. Indeed it has been reported that in other pathologies with strong inflammatory and oxidative profiles such as acute on chronic liver failure, a condition with many similarities with sepsis, endogenous albumin can reach higher HNA2 absolute values (up to $30 \%$ ) than those detected in our work (Clària et al., 2016).

While the oxidative fractions of albumin are related to the intensity of stress, the absolute concentrations of each fraction in $\mathrm{g} / \mathrm{L}$ indicate how much albumin was available to react with ROS and how much of albumin already underwent this reaction, reversibly or irreversibly. Since ROS can induce chain reactions, the oxidation of albumin indicates that other molecules are substantially spared from the oxidative cascade. In previous studies (Bourdon and Blache, 2001; Lang et al., 2004; Liu et al., 2012; Vincent et al., 2016), we found an indication of the benefits of albumin administration in septic shock patients, where the intensity of oxidative stress was higher. It is possible that the sparing action of albumin, which is proportional to its concentration, may improve outcome in a fraction of septic shock patients, but this finding needs confirmation.

In conclusion, the data presented here suggest that the oxidation status of albumin in patients with sepsis is related to the time of exposure to oxidative stress (differences between day 1 and day 7). After 7 days of treatment, lower SOFA scores correlate with higher albumin antioxidant capacity. The trend toward a positive effect of albumin treatment, while not statistically significant, warrants further investigation.

\section{DATA AVAILABILITY STATEMENT}

Data is available upon request, to be examined and accepted by the Steering Committee of ALBIOS trial.

\section{ETHICS STATEMENT}

This study was reviewed and approved by each Ethics Committee of the 100 centers included in the ALBIOS trial. The patients/ participants provided their written informed consent to participate in this study.

\section{AUTHOR CONTRIBUTIONS}

MBo, JM, RL, and LGa have made substantial contributions to the conception and design of the work. $\mathrm{AP}, \mathrm{FVq}, \mathrm{MBu}$, FVs, DN, RB, CS, FR, LGi, MM, and IP contributed to the acquisition of data. MBo, JM, AP, SM, RL, and LGa contributed to the analysis and interpretation of data. MBo, JM, MP, FM, MC, PC, RL, MQ, and LGa have drafted the work or substantively revised it. All authors have approved the submitted version (and any substantially modified version that involves the author's contribution to the study), and have agreed both to be personally accountable for the author's own contributions and to ensure 
that questions related to the accuracy or integrity of any part of the work, even ones in which the author was not personally involved, are appropriately investigated, resolved, and the resolution documented in the literature.

\section{FUNDING}

The Albumin Italian Outcome Sepsis trial was funded by a grant from the Italian Medicines Agency (AIFA, grant FARM6JS3R5, 2006).

\section{REFERENCES}

Anraku, M., Chuang, V. T. G., Maruyama, T., and Otagiri, M. (2013). Redox properties of serum albumin. Biochim. Biophys. Acta 1830, 5465-5472. doi: 10.1016/j.bbagen.2013.04.036

Arroyo, V., García-Martinez, R., and Salvatella, X. (2014). Human serum albumin, systemic inflammation, and cirrhosis. J. Hepatol. 61, 396-407. doi: 10.1016/j. jhep.2014.04.012

Barle, H., Januszkiewicz, A., Hållström, L., Essén, P., McNurlan, M. A., Garlick, P. J., et al. (2002). Albumin synthesis in humans increases immediately following the administration of endotoxin. Clin. Sci. (Lond.) 103, 525-531. doi: 10.1042/ CS20020181

Bar-Or, D., Bar-Or, R., Rael, L. T., Gardner, D. K., Slone, D. S., and Craun, M. L. (2005). Heterogeneity and oxidation status of commercial human albumin preparations in clinical use. Crit. Care Med. 33, 1638-1641. doi: 10.1097/01. CCM.0000169876.14858.91

Bavunoglu, I., Genc, H., Konukoglu, D., Cicekci, H., Sozer, V., Gelisgen, R., et al. (2016). Oxidative stress parameters and inflammatory and immune mediators as markers of the severity of sepsis. J. Infect. Dev. Ctries. 10, 1045-1052. doi: 10.3855/jidc.7585

Bourdon, E., and Blache, D. (2001). The importance of proteins in Defense Against oxidation. Antioxid. Redox Signal. 3, 293-311. doi: 10.1089/ 152308601300185241

Caironi, P., Masson, S., Mauri, T., Bottazzi, B., Leone, R., Magnoli, M., et al. (2017). Pentraxin 3 in patients with severe sepsis or shock: the ALBIOS trial. Eur. J. Clin. Investig. 47, 73-83. doi: 10.1111/eci.12704

Caironi, P., Tognoni, G., Masson, S., Fumagalli, R., Pesenti, A., Romero, M., et al. (2014). Albumin replacement in patients with severe sepsis or septic shock. N. Engl. J. Med. 370, 1412-1421. doi: 10.1056/NEJMoa1305727

Clària, J., Stauber, R. E., Coenraad, M. J., Moreau, R., Jalan, R., Pavesi, M., et al. (2016). Systemic inflammation in decompensated cirrhosis: characterization and role in acute-on-chronic liver failure. Hepatology 64, 1249-1264. doi: 10.1002/hep.28740

Colombo, G., Clerici, M., Giustarini, D., Rossi, R., Milzani, A., and Dalle-Donne, I. (2012). Redox albuminomics: oxidized albumin in human diseases. Antioxid. Redox Signal. 17, 1515-1527. doi: 10.1089/ars.2012.4702

Das, S., Maras, J. S., Hussain, M. S., Sharma, S., David, P., Sukriti, S., et al. (2017). Hyperoxidized albumin modulates neutrophils to induce oxidative stress and inflammation in severe alcoholic hepatitis. Hepatology 65, 631-646. doi: $10.1002 /$ hep. 28897

Galley, H. F. (2011). Oxidative stress and mitochondrial dysfunction in sepsis. Br. J. Anaesth. 107, 57-64. doi: 10.1093/bja/aer093

Hayashi, T., Era, S., Kawai, K., Imai, H., Nakamura, K., Onda, E., et al. (2000). Observation for redox state of human serum and aqueous humor albumin from patients with senile cataract. Pathophysiology 6, 237-243. doi: 10.1016/ S0928-4680(99)00022-X

Huet, O., Obata, R., Aubron, C., Spraul-Davit, A., Charpentier, J., Laplace, C., et al. (2007). Plasma-induced endothelial oxidative stress is related to the severity of septic shock. Crit. Care Med. 35, 821-826. doi: 10.1097/01. CCM.0000257464.79067.AF

Kumar, S., Gupta, E., Kaushik, S., Kumar Srivastava, V., Mehta, S. K., and Jyoti, A. (2018). Evaluation of oxidative stress and antioxidant status: correlation with the severity of sepsis. Scand. J. Immunol. 87:e12653. doi: 10.1111/ sji. 12653

\section{ACKNOWLEDGMENTS}

We would like to thank the participating patients.

\section{SUPPLEMENTARY MATERIAL}

The Supplementary Material for this article can be found online at: https://www.frontiersin.org/articles/10.3389/fphys.2021.682877/ full\#supplementary-material

Lang, J. D., Figueroa, M., Chumley, P., Aslan, M., Hurt, J., Tarpey, M. M., et al. (2004). Albumin and hydroxyethyl starch modulate oxidative inflammatory injury to vascular endothelium. Anesthesiology 100, 51-58. doi: 10.1097/00000542-200401000-00012

Liu, S.-Y., Chen, C.-L., Yang, T.-T., Huang, W.-C., Hsieh, C.-Y., Shen, W.-J., et al. (2012). Albumin prevents reactive oxygen species-induced mitochondrial damage, autophagy, and apoptosis during serum starvation. Apoptosis 17, 1156-1169. doi: 10.1007/s10495-012-0758-6

Mantzarlis, K., Tsolaki, V., and Zakynthinos, E. (2017). Role of oxidative stress and mitochondrial dysfunction in Sepsis and potential therapies. Oxidative Med. Cell. Longev. 2017:5985209. doi: 10.1155/2017/5985209

Masson, S., Caironi, P., Fanizza, C., Carrer, S., Caricato, A., Fassini, P., et al. (2015). Sequential N-terminal pro-B-type natriuretic peptide and highsensitivity cardiac troponin measurements During albumin replacement in patients With severe Sepsis or septic shock. Crit. Care Med. 44, 707-716. doi: 10.1097/CCM.0000000000001473

Matsuyama, Y., Terawaki, H., Terada, T., and Era, S. (2009). Albumin thiol oxidation and serum protein carbonyl formation are progressively enhanced with advancing stages of chronic kidney disease. Clin. Exp. Nephrol. 13, 308-315. doi: 10.1007/s10157-009-0161-y

Mauri, T., Bellani, G., Patroniti, N., Coppadoro, A., Peri, G., Cuccovillo, I., et al. (2010). Persisting high levels of plasma pentraxin 3 over the first days after severe sepsis and septic shock onset are associated with mortality. Intensive Care Med. 36, 621-629. doi: 10.1007/s00134-010-1752-5

Mera, K., Anraku, M., Kitamura, K., Nakajou, K., Maruyama, T., and Otagiri, M. (2005). The structure and function of oxidized albumin in hemodialysis patients: its role in elevated oxidative stress via neutrophil burst. Biochem Biophys. Res. Commun. 334, 1322-1328. doi: 10.1016/j.bbrc.2005.07.035

Nagar, H., Piao, S., and Kim, C.-S. (2018). Role of mitochondrial oxidative stress in Sepsis. Acute Crit. Care 33, 65-72. doi: 10.4266/acc.2018.00157

Nakashima, F., Shibata, T., Kamiya, K., Yoshitake, J., Kikuchi, R., Matsushita, T., et al. (2018). Structural and functional insights into S-thiolation of human serum albumins. Sci. Rep. 8:932. doi: 10.1038/s41598-018-19610-9

Oettl, K., and Marsche, G. (2010). Redox state of human serum albumin in terms of cysteine-34 in health and disease. Methods Enzymol. 474, 181-195. doi: 10.1016/S0076-6879(10)74011-8

Park, D. W., and Zmijewski, J. W. (2017). Mitochondrial dysfunction and immune cell metabolism in Sepsis. Infect. Chemother. 49, 10-21. doi: 10.3947/ic.2017.49.1.10

Quinlan, G. J., Margarson, M. P., Mumby, S., Evans, T. W., and Gutteridge, J. M. (1998). Administration of albumin to patients with sepsis syndrome: a possible beneficial role in plasma thiol repletion. Clin. Sci. (Lond.) 95, 459-465. doi: 10.1042/CS19980191

Rhodes, A., Evans, L. E., Alhazzani, W., Levy, M. M., Antonelli, M., Ferrer, R., et al. (2017). Surviving Sepsis campaign: international guidelines for Management of Sepsis and Septic Shock: 2016. Intensive Care Med. 43, 304-377. doi: 10.1007/s00134-017-4683-6

Salvemini, D., and Cuzzocrea, S. (2002). Oxidative stress in septic shock and disseminated intravascular coagulation. Free Radic. Biol. Med. 33, 1173-1185. doi: 10.1016/S0891-5849(02)00961-9

Singer, M., Deutschman, C. S., Seymour, C. W., Shankar-Hari, M., Annane, D., Bauer, M., et al. (2016). The third international consensus definitions for Sepsis and septic shock (Sepsis-3). JAMA 315, 801-810. doi: 10.1001/jama.2016.0287

Soejima, A., Matsuzawa, N., Hayashi, T., Kimura, R., Ootsuka, T., Fukuoka, K., et al. (2004). Alteration of redox state of human serum 
albumin before and after hemodialysis. Blood Purif. 22, 525-529. doi: $10.1159 / 000082524$

Vasques, F., Duscio, E., Romitti, F., Pasticci, I., Caironi, P., Meessen, J., et al. (2018). Septic shock-3 vs 2: an analysis of the ALBIOS study. Crit. Care 22:237. doi: 10.1186/s13054-018-2169-8

Víctor, V. M., Espulgues, J. V., Hernández-Mijares, A., and Rocha, M. (2009). Oxidative stress and mitochondrial dysfunction in sepsis: a potential therapy with mitochondria-targeted antioxidants. Infect. Disord. Drug Targets 9, 376-389. doi: 10.2174/187152609788922519

Vincent, J. L., De Backer, D., and Wiedermann, C. J. (2016). Fluid management in sepsis: The potential beneficial effects of albumin. J. Crit. Care 35, 161-167. doi: 10.1016/j.jcrc.2016.04.019

Vincent, J.-L., Russell, J. A., Jacob, M., Martin, G., Guidet, B., Wernerman, J., et al. (2014). Albumin administration in the acutely ill: what is new and where next? Crit. Care 18:231. doi: 10.1186/cc13991

Conflict of Interest: MC, FM, and AP are full-time employees of Grifols, a manufacturer of plasma derivatives. Grifols had no access to clinical data from
ALBIOS and did not perform statistical analyses of data, but only provided editorial support. Publication costs of this manuscript were sustained by Grifols. LG and PC received honoraria from Grifols.

Publisher's Note: All claims expressed in this article are solely those of the authors and do not necessarily represent those of their affiliated organizations, or those of the publisher, the editors and the reviewers. Any product that may be evaluated in this article, or claim that may be made by its manufacturer, is not guaranteed or endorsed by the publisher.

Copyright () 2021 Bonifazi, Meessen, Pérez, Vasques, Busana, Vassalli, Novelli, Bernasconi, Signori, Masson, Romitti, Giosa, Macri, Pasticci, Palumbo, Mota, Costa, Caironi, Latini, Quintel and Gattinoni. This is an open-access article distributed under the terms of the Creative Commons Attribution License (CC BY). The use, distribution or reproduction in other forums is permitted, provided the original author(s) and the copyright owner(s) are credited and that the original publication in this journal is cited, in accordance with accepted academic practice. No use, distribution or reproduction is permitted which does not comply with these terms. 